\title{
Space-station experiment deepens antimatter enigma
}

First results from the Alpha Magnetic Spectrometer fall short of evidence for dark matter.

\author{
Eugenie Samuel Reich
}

03 April 2013

The Alpha Magnetic Spectrometer has seen extra antimatter particles at high energies.

Some of the International Space Station's most anticipated science results are in, but their interpretation — which hints at a darkmatter detection — is likely to be debated by physicists for years to come.

Principal investigator Samuel Ting presented the first data today from the Alpha Magnetic Spectrometer (AMS), a US\$1.5-billion cosmic-ray detector fixed to the outside of the station. In a talk at CERN, the particle-physics facility near Geneva, Switzerland, Ting told physicists that the mission has confirmed data from the European satellite Payload for Antimatter Matter Exploration and Lightnuclei Astrophysics (PAMELA) and NASA's Fermi Gamma-ray Space Telescope showing that something in the Galaxy is spewing out many more positrons — the antimatter counterparts of electrons — than can be accounted for from known astrophysical sources.

Yet the spectrum of this antimatter excess is far from a smoking gun for models in which the extra positrons are generated through the annihilation of dark-matter particles colliding with each other. "The detailed interpretation of our data probably will have many theories," says Ting.

\section{No definitive picture}

Comprising a giant magnet and eight particle trackers, the AMS was launched in 2011 after a 17-year campaign by Ting, a physicist and Nobel laureate at the Massachusetts Institute of Technology (MIT) in Cambridge, to place an antimatter detector in space. Now, in a paper to be published in Physical Review Letters, Ting and his team say that over the mission's first 18 months of operations, they observed some 6.8 million positrons and electrons, at energies up to 350 gigaelectronvolts (GeV). These are higher-energy events than PAMELA and Fermi were able to see, but still less than half the energy that AMS will be sensitive to over its 15-year lifetime. The detection accuracy of the AMS falls with increasing energy, and Ting and his team have chosen not to release data at energies above $350 \mathrm{GeV}$ because the results do not yet have enough statistical significance to give a definitive picture.

Still, the spectrum looks promising. It shows a rise of positrons with energy that begins to flatten off at energies above $250 \mathrm{GeV}$. If the signal is caused by dark matter, the number of positrons should rise and then drop again around the mass of the dark-matter particle, which cannot produce positrons more energetic than itself. The data provide "a tantalizing hint of something exciting", says Michael Turner, a theoretical cosmologist at the University of Chicago in Illinois.

An alternative theory is that the antimatter could be emanating from pulsars, rotating superdense stars in the Galaxy whose properties are not perfectly understood. "I personally think the pulsar explanation is more viable now," says Dan Hooper, a theorist at Fermilab in Batavia, Illinois. He is struck by the absence, so far, of a sharp decrease in antimatter at higher energies.

But Ting says that the AMS collects antimatter uniformly from all over the sky, which does not seem to indicate that specific astrophysical point sources, such as pulsars, are the cause.

One problem, raised by Peter Fisher, an AMS collaborator at MIT, is that there is still no consensus about what the expected background rate of antimatter in the Galaxy should be - and, therefore, about how much of an excess the AMS is seeing. "It's like playing blind-man's bluff," he says. Despite the anticipation that has built around this announcement, he and Ting now say that physicists will have to wait another couple of years for the AMS to release its higher-energy data to know whether the excess is due to dark matter, pulsars or something else.

Nature | doi:10.1038/nature.2013.12718 